\title{
Non-ablative radio frequency for the treatment of androgenetic alopecia
}

\author{
Yimei Tan ${ }^{1}$, Liu Wei ${ }^{2}$, Yiyi Zhang ${ }^{1}$, Andy Goren ${ }^{3,4}$, John McCoy³, Andrija Stanimirovic ${ }^{5}$, Torello Lotti ${ }^{4}$, Maja Kovacevic ${ }^{6 凶}$
}

\begin{abstract}
Introduction: Medical treatment of androgenetic alopecia (AGA) is mainly limited to pharmacological and surgical interventions. Patients' desire for noninvasive and non-systemic treatments has accelerated research into medical devices that can promote hair growth. Low-level laser therapy (LLLT) was the first such device. However, its success has been limited by contradictory and often controversial efficacy claims. Work previously performed in animal models of AGA has demonstrated the viability of the wound repair mechanism as a potential treatment modality. This study therefore explores the use of a non-ablative radio frequency (RF) device in the treatment of AGA.
\end{abstract}

Methods: A single blinded study compared a non-ablative RF device versus a sham device in 24 men with AGA. Each subject received four treatments over the 12-week study.

Results: In this preliminary study of 24 AGA patients treated with a novel RF device, we demonstrated that $54 \%$ showed a clinical response. Furthermore, among patients that underwent four or more treatment sessions, $40 \%$ experienced a $30 \%$ or more increase in hair counts compared to baseline.

Conclusions: If validated in a larger cohort, non-ablative RF may prove to be an important clinical tool in the treatment of AGA.

Keywords: radio frequency, androgenetic alopecia, treatment

Received: 24 August 2019| Returned for modification: 9 November 2019|Accepted: 13 November 2019

\section{Introduction}

Androgenetic alopecia (AGA) affects up to $50 \%$ of the population by age 60 (1). To date, the US Food and Drug Administration (FDA) has only approved two drugs for the treatment of AGA: topical minoxidil and oral finasteride. In addition to pharmacological interventions and surgical hair restoration, low-level laser therapy (LLLT) devices for the treatment of AGA have been introduced to the market. The efficacy of these devices has been subject to controversy due to a lack of quality evidence (2), and so there is a large unmet medical need for a non-invasive treatment for AGA.

Several researchers have independently shown the role of wound healing in hair regrowth. Chuong reported that wound healing induces new hair growth in mice (3). Ansell et al. reported acceleration of wound healing during the hair anagen phase (4). Sun et al. reported a case of new hair growth on a bald scalp following wound repair (5). In addition, Dhurat et al. demonstrated that the use of microneedling in combination with minoxidil was superior to minoxidil mono-therapy for the treatment of AGA (6). Although it is not clear whether the microneedling enhanced minoxidil permeability or sulfotransferase enzyme induction, it nevertheless serves as additional human evidence for hair follicle regeneration through injury (6).

Non-ablative RF is a common mode of treatment for skin wrinkles and laxity (7). The treatment induces dermal injury by heat generated under the skin (8). We hypothesized that using a specially adapted non-ablative RF device to deliver treatment to the scalp will be an effective treatment for AGA.

\section{Methods}

Twenty-four otherwise healthy male subjects were recruited from a dermatology clinic. Subjects were diagnosed at baseline with
Norwood stage 3 or above. The average age of the subjects was 29.5 years. The study was approved by the hospital ethics committee (Hairmore Hospital Group, Beijing, China) and all subjects provided informed consent prior to beginning the trial. A specially adapted fractional non-ablative RF device (Innogen, Tel Aviv, Israel) was used for application of the treatment. The device generates a RF of $460 \mathrm{KHz}$ with a power output of $12 \mathrm{~W}$ and $62 \mathrm{~mJ} / \mathrm{pin}$.

The study design was a single-blinded placebo-controlled study. Five subjects were assigned to the placebo (sham device) group and 19 to the active group. The placebo group received the same treatment with the RF device except that the device was powered off. Subjects received an average of four treatment sessions administered every 3 weeks. Each session lasted for approximately 5 minutes at high power settings. The placebo group was intentionally small due to the small size of the study and the known placebo response of hair growth to placebo drug trials; that is, less than a $13.7 \%$ increase in hair counts for the topical minoxidil vehicle (9). An independent two-sided samples t-test (MedCalc v18.2.1) was used to determine the significance of average increase in hair counts from baseline in the treatment group versus the placebo group. At baseline, a $1 \mathrm{~cm}^{2}$ target area at the vertex of the scalp was shaved on each subject. A tattoo was administered at the center of the target area. A photo-trichogram of the target area was taken (FotoFinder, Germany) and at the end of the study, a photo-trichogram of the target area was repeated (FotoFinder, Germany). Hair counts were performed manually by a medical resident.

\section{Results}

Subjects completed the study with an average of 3.63 sessions. The average increase in hair counts from baseline in the treatment group was higher than the placebo group $22.8 \%$ vs $9.6 \%(p=0.08)$. 
The results are tabulated in Table 1 and shown in Fig. 1. Although the significance did not reach the $p=0.05$ level, we believe the effect is of clinical importance because only one of the five subjects in the placebo group had a clinical response; that is, an increase in hair counts greater than $13.7 \%$ compared to baseline (9). None of the subjects reported treatment-related adverse events; however, the smell from tissue damage during therapy was of concern to most (circa $75 \%$ ) of the subjects in the treatment arm.

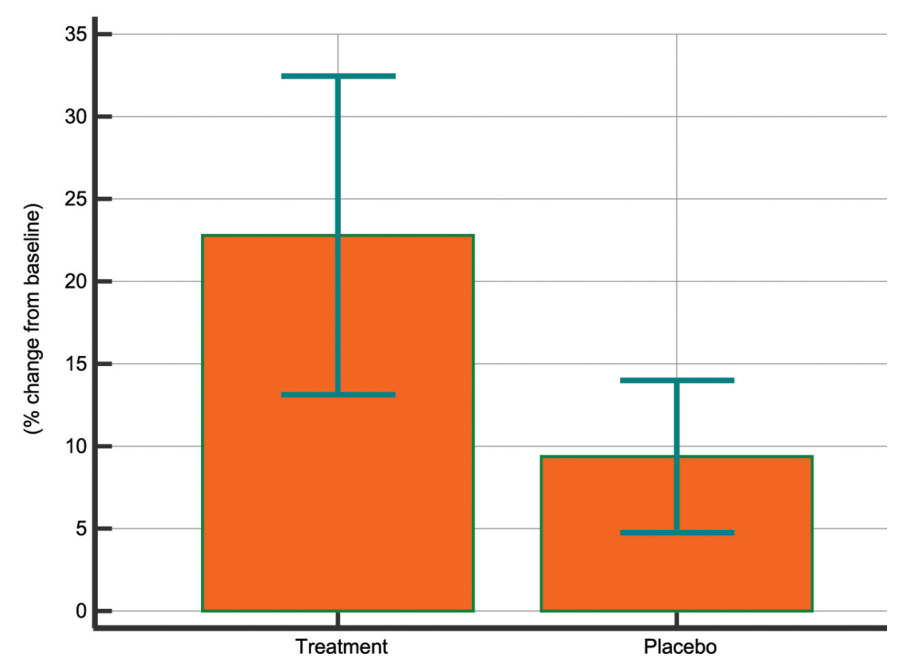

Figure 1 | Average percent change in target area hair counts (TAHC) after treatment with a non-ablative radio frequency device.

\section{Discussion}

Previous studies on AGA preformed using animal models demonstrated the viability of the wound repair mechanism as a potential treatment modality for AGA (3-5). Moreover, a pilot study on 100 cases of mild to moderate AGA comparing the effectiveness of $5 \%$ minoxidil lotion only and $5 \%$ minoxidil lotion combined with microneedling treatment demonstrated that microneedling with minoxidil was superior to minoxidil monotherapy for the treatment of AGA (6). Non-ablative RF can similarly induce dermal injury by heat generated under the skin (8). To that end, we explored the use of a non-ablative RF device to induce scalp tissue wound healing mechanisms for hair regrowth. Non-ablative RF is appropriate for all skin types and hair colors, and we are not aware of any potential contradictions to the procedure. In our study, we observed significant hair regrowth in the treatment arm compared to the placebo arm.

We chose a similar protocol used for skin rejuvenation, four sessions in total performed 3 weeks apart. One limitation of this study was that not all the patients in the treatment arm received the minimum recommended treatments; for example, Subject 3 only received two treatments total. Nevertheless, we observed a striking difference between the treatment arm and the sham device. In addition, all the patients in the placebo arm received the recommended minimum number of treatments. Although the sample size of the study was small, it demonstrates that non-ablative RF could potentially provide a non-invasive method to stimulate hair regrowth as an additional treatment modality for alopecia. A larger study is needed to confirm these results.

\section{Conclusions}

Wound healing is associated with hair regrowth in various animal models. Microneedling of scalp tissue presents promising evidence for a similar mechanism for inducing hair regrowth in humans. Here we demonstrate that a non-ablative RF device can be an effective treatment for AGA.

Table 1 | Percent change in target area hair counts (TAHC) at baseline versus after treatment with non-ablative radio frequency device. Data are tabulated first according to treatment arm and then by percent increase in TAHC.

\begin{tabular}{|c|c|c|c|c|c|c|}
\hline ID & Age & Sessions & Arm & Baseline hair count & Post-treatment hair count & $\%$ change \\
\hline 1 & 31 & 4 & Treatment & 19 & 31 & 63.16 \\
\hline 2 & 28 & 6 & Treatment & 16 & 26 & 62.50 \\
\hline 3 & 26 & 2 & Treatment & 16 & 23 & 43.75 \\
\hline 4 & 25 & 3 & Treatment & 18 & 25 & 38.89 \\
\hline 5 & 30 & 4 & Treatment & 12 & 16 & 33.33 \\
\hline 6 & 44 & 6 & Treatment & 6 & 8 & 33.33 \\
\hline 7 & 31 & 3 & Treatment & 18 & 23 & 27.78 \\
\hline 8 & 28 & 3 & Treatment & 20 & 25 & 25.00 \\
\hline 9 & 30 & 2 & Treatment & 22 & 27 & 22.73 \\
\hline 10 & 31 & 4 & Treatment & 21 & 25 & 19.05 \\
\hline 11 & 34 & 2 & Treatment & 16 & 19 & 18.75 \\
\hline 12 & 24 & 4 & Treatment & 19 & 22 & 15.79 \\
\hline 13 & 28 & 3 & Treatment & 22 & 25 & 13.64 \\
\hline 14 & 32 & 4 & Treatment & 23 & 25 & 8.70 \\
\hline 15 & 28 & 7 & Treatment & 23 & 25 & 8.70 \\
\hline 16 & 34 & 2 & Treatment & 19 & 20 & 5.26 \\
\hline 17 & 32 & 2 & Treatment & 20 & 21 & 5.00 \\
\hline 18 & 24 & 2 & Treatment & 23 & 24 & 4.35 \\
\hline 19 & 24 & 6 & Treatment & 24 & 20 & -16.67 \\
\hline 20 & 20 & 4 & Placebo & 20 & 23 & 15.00 \\
\hline 21 & 26 & 4 & Placebo & 21 & 23 & 9.52 \\
\hline 22 & 24 & 4 & Placebo & 22 & 24 & 9.09 \\
\hline 23 & 50 & 4 & Placebo & 23 & 25 & 8.70 \\
\hline 24 & 29 & 4 & Placebo & 22 & 23 & 4.55 \\
\hline
\end{tabular}

\section{References}

1. Wang TL, Zhou C, Shen YW, Wang XY, Ding XL, Tian S, et al. Prevalence of androgenetic alopecia in China: a community-based study in six cities. $\mathrm{Br} J$ Dermatol. 2010;162:843-7.
2. van Zuuren EJ, Fedorowicz Z, Schoones J. Interventions for female pattern hair loss. Cochrane Database Syst Rev. 2016;2016:CDoo7628. 
3. Chuong CM. Regenerative biology: new hair from healing wounds. Nature. 2007; 447:265-6.

4. Ansell DM, Kloepper JE, Thomason HA, Paus R, Hardman MJ. Exploring the "hai growth-wound healing connection": anagen phase promotes wound re-epithelialization. J Invest Dermatol. 2011;131:518-28.

5. Sun ZY, Diao JS, Guo SZ, Yin GQ. A very rare complication: new hair growth around healing wounds. Int Med Res. 2009;37:583-6.

6. Dhurat R, Sukesh M, Avhad G, Dandale A, Pal A, Pund P. A randomized evaluator blinded study of effect of microneedling in androgenetic alopecia: a pilot study. Int J Trichology. 2013;5:6-11.
7. Araújo AR, Soares VP, Silva FS, Moreira Tda S. Radiofrequency for the treatment of skin laxity: myth or truth. An Bras Dermatol. 2015;90:707-21.

8. Gold M. Update on tissue tightening. J Clin Aesthet Dermatol. 2010;3:36-41.

9. McCoy J, Goren A, Kovacevic M, Shapiro J. Minoxidil dose response study in female pattern hair loss patients determined to be non-responders to $5 \%$ topical minoxidil. J Biol Regul Homeost Agents. 2016;30:1153-5. 